\title{
Paper
}

\section{Optimization of Clutch Drum Heat Treatment Processing Condition for Automobile AT using "Robust Design" $\sim$ Reduction of variation in teeth deformation amount by optimizing clutch drum welding process condition}

\author{
EIJI TOMA* ${ }^{* \dagger}$ Member
}

(Received April 18, 2018, revised July 1, 2018)

\begin{abstract}
A lot of parts for transmitting power are built in the inside of the automatic transmission of the automobile. Clutch pack parts having important functions related to speed change adopt machining and heat treatment processes as the main manufacturing method from the viewpoint of high productivity and demanding dimensional accuracy requirement. In the welding and heat treatment process, quality defects such as variation in dimensional accuracy due to thermal deformation of the drum teeth portion occur. In solving this quality problem, we optimized processing conditions by using quality engineering method (Taguchi method), realized quality assurance and low cost by reducing variation in teeth deformation amount.
\end{abstract}

Keywords: Robust design, static characteristic evaluation, SN ratio, factor effect diagram, reliability confirmation

\section{Introduction}

A number of components (clutch disk, seal ring, return spring, piston, inner snap ring, cancel cover, etc.) for transmitting power are incorporated in the automobile automatic transmission (Fig. 1). Among them, clutch pack parts (Fig. 2), which have important functions related to speed change, adopt machining and heat treatment processing steps as the main manufacturing method from the viewpoint of high productivity and demanding dimensional accuracy. Fig. 2 shows an overview of the power transmission mechanism of the clutch pack. By supplying hydraulic pressure to the clutch piston, the disc and the mating plate are pressed, and when they are tightened, power is transmitted. When the hydraulic pressure is released, the piston is pushed back by the return spring, and it becomes released state. So that the torque transmitted between the two members can be controlled according to the magnitude of the clutch pressure [8].

\section{Research Purpose}

Fig. 3 shows a schematic view of joining the clutch drum (drum) and the shaft hub (hub). The problem of this research is that in the automatic welding process (E.B.W; Electron Beam Welding) of the drum and the shaft hub in the heat treatment process, there are variations in dimensional accuracy due to deformation of the tooth width shape

\footnotetext{
* Corresponding Author: toma-e@ tomakomai-ct.ac.jp

$\dagger$ National Institute of Technology, Tomakomai College

Tomakomai, Nishikioka 443, 059-1275 Hokkaido, Japan
}

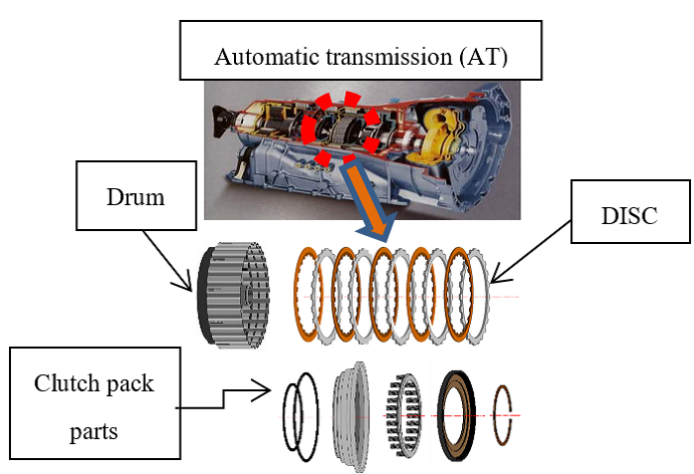

Figure 1: Components in clutch drum

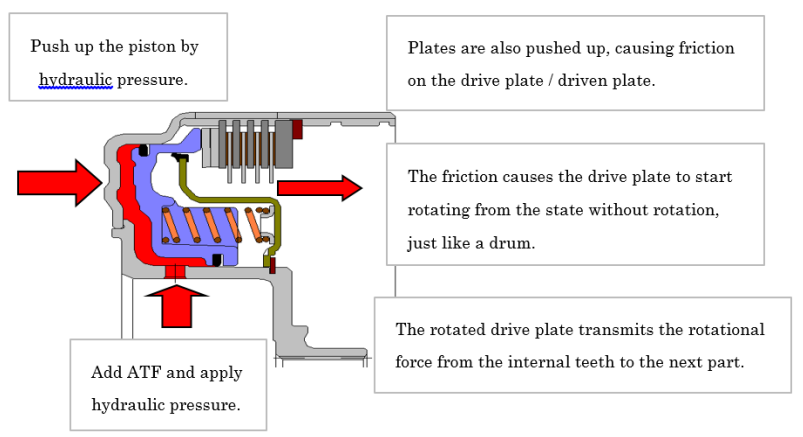

Figure 2: Clutch pack power transmission mechanism

of the drum spline portion and defects in the quality function is generated.

In a general engineering process, there may be variations 


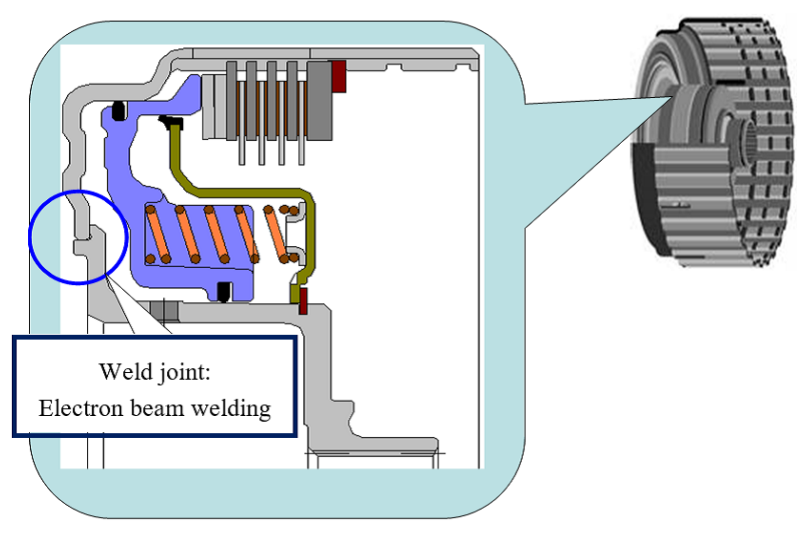

Figure 3: Weld joint of drum and hub

in various conditions, such as variations in materials and production machinery. Optimization of robust product processing conditions that can withstand these variations is an important issue. Products manufactured by pursuing optimum values at the marginal theoretical value in constraint conditions in "Manufacturing at the production site" are susceptible to variations in conditions.

As a result, the trade-off problem of increasing the defect rate and lowering the process capability (ability to produce the product within a specified standard limit) will develop. In order to solve these quality problems efficiently, practical implementation of "robust design" which is an analysis evaluation technology of robust and optimum processing conditions is extremely effective [3] [4].

The purpose of this research is to optimize the clutch drum welding processing condition using this method, to realize efficient quality assurance and cost reduction at the production site by reducing variation in tooth width deformation amount and improving process capability.

\section{Outline of Heat Treatment Process}

The main manufacturing process of the clutch pack part is developed into assembly process of pack functional parts through heat treatment steps of welding joining (EBW) between the drum and the hub and surface reforming treatment (GNP; Gas Nitriding Processing ) [1].

Fig. 4 shows the outline of welding process equipment (EBW). The reason why the welding method is adopted for joining the drum and the hub is that the cost merit is larger than the integrated cutting processing of the drum and the hub. However, despite the cost merit, at present it is the fact that the inspection process of the product shape and dimensions is indispensable in order to guarantee quality assurance, which is an important issue that the production site has.

The EBW is a welding method that uses energy of a highspeed electron beam generated in a vacuum as a heat source. There is a characteristic that penetration becomes very deep with respect to the width of welding. It is not suitable for large or long structures because it requires a vacuum chamber for containing an electron gun and a welding base metal. In the control mechanism, several setting modes such as the radiation / convergence current value of the electron beam,

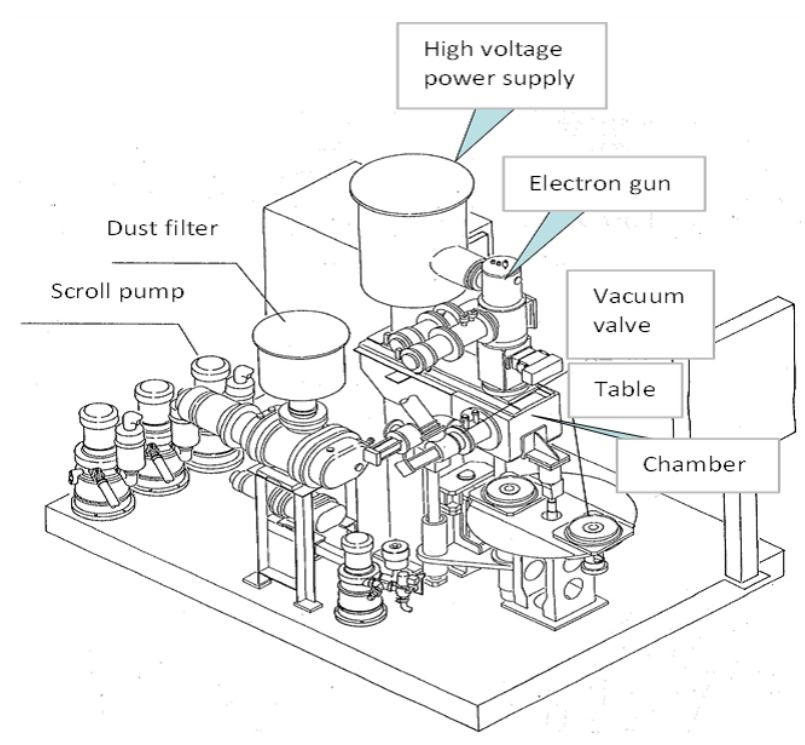

Figure 4: Outline of welding machine

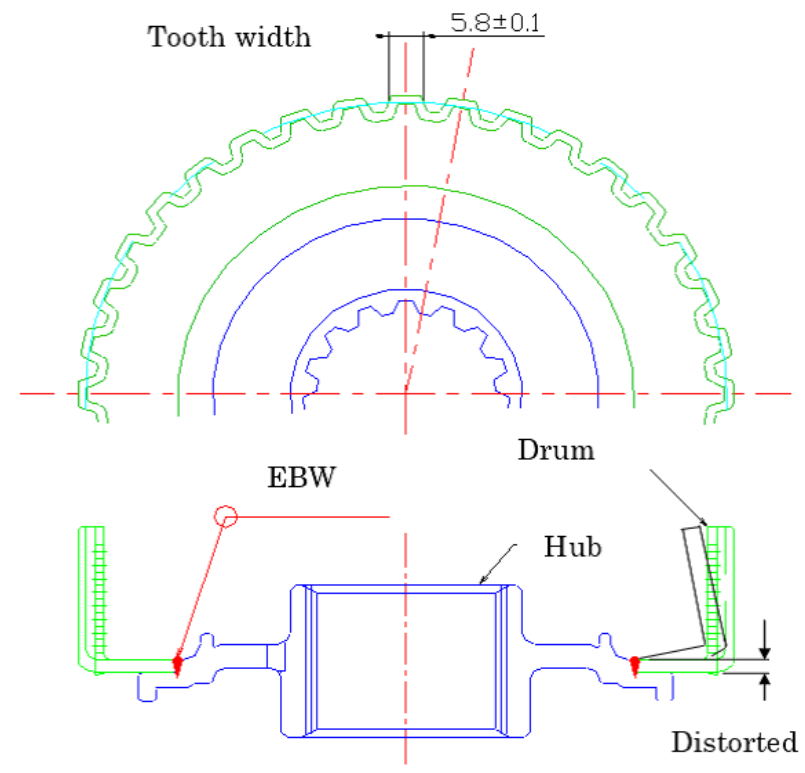

Figure 5: After the welding is distorted

the machining table rotation speed, and the beam radiation angle are incorporated. As shown in Fig. 5, distortion occurs in the drum due to the influence of penetrating thermal energy applied at the time of welding the electron beam of the drum and the hub, causing quality problems such as deformation of the drum tooth portion and deviation of dimensional specification. In particular, variations in the drum tooth width dimension occur, and the process capability of the standard size becomes insufficient [2].

In the shipment inspection, it is necessary to perform a total inspection to ensure the quality, resulting in an increase in the manufacturing cost.

\section{Application of "Robust design"}

In order to simultaneously ensure quality and reduce manufacturing cost, "Parameter design" which is the main systematic method of "Taguchi method (Robust design)" was 
applied and optimum welding processing conditions were extracted.

4.1 Concept of "Robust design" In the conventional design method, development is conducted while testing the target function system under standard conditions. When it is found that the function can be obtained under the standard condition, it is tested according to some conditions, for example by changing the use environment or load test. The problem is to adjust to have aiming function while changing design constraints when a case occurs that does not satisfy the target function when testing with changing various conditions Adjustment (tuning) is not designed to suppress the interaction between design parameters and signals and noise, so it tends to be infinite design work with whack-amole.

On the other hand, robust design with Taguchi method is to do using two-step design method. As a procedure, first, after reducing the variation of the function, it adapts to the target value of the target function and the target curve. In the Taguchi method, three tools are used as a concrete method (engineering means): 1: Orthogonal Table, 2: SN Ratio, 3: Basic Function (Ideal Function) Setting. To efficiently design products that work correctly under various conditions of using these tools is to do robust design with quality engineering (Taguchi method). The reasons for using these three tools are explained below [5].

4.1.1 Orthogonal Table One of the reasons to use the orthogonal table is to study the optimum design as far as possible to maximize development efficiency and to incorporate the idea of front loading. Another reason is to evaluate reliability against downstream conditions. That is, since the optimum design in the upstream is optimal without causing quality problems even in the downstream, only the main effect is allocated to the orthogonal table, and the main effect of the control factor which the designer can freely select and implement is additive. It is because it is necessary to check whether or not.

4.1.2 SN Ratio The SN Ratio (SNR) is a signal-tonoise ratio, and it is widely used in many fields such as communication engineering, electrical engineering, communication, recording and playback. Even if the signal is large, it can be said that it is not good as a function if the proportion of noise is large.

The reason for using this SNR will be described. First of all, various use conditions for a certain system in the market are "signal" or "noise". Neither "noise" nor "signal" is factor that cannot be controlled by the designer. "Signal" is a general condition that a user uses a product, and it is necessary to investigate its function widely as far as possible in the range of the signal factor as much as possible among various assumed usage conditions. On the other hand, "noise" is a factor that affects characteristic values due to variations that are different from signal factors, and it is necessary to grasp the conditions. The reason for this kind of separation is to evaluate whether or not the product functions correctly against the use conditions that cannot be freely made by the designer. The fact that the SNR is high means that the influence on the variations of product functions caused by noise is small with respect to various assumed signal factors, which means that it exhibits the expected product function.

4.1.3 Basic Function (Ideal Function) Setting Robust design expresses the function of the created technology by using the basic function of the product as an ideal function that the inputs and outputs are linear with each other. The difference between the ideal function (or target function) and the actual characteristic value is considered as functionality and evaluated by the SNR. Conventionally, after selecting the system (idea) that realizes the aimed function first, the functional design was performed, and then the design (suppressing variation) was performed. It is difficult to suppress the influence of the variation and the interaction when trying to compress the variation of the function under various conditions after obtaining the target function under the standard condition.

In quality engineering, functional design is performed after functional design. First of all, by reducing the variation of the function and then designing the function so as to match the target value or the target curve, efforts such as variation compression are made more efficient. The function of the system is created by conversion of energy.

The robustness of system function is to think "ideal function" of energy conversion. In order to realize the ideal function, since the individual characteristics of the system are involved, it is necessary to devise how to set the ideal function.

\section{Parameter design}

Parameter design is a method of improving robustness by generating deliberate variations with noise factors among combinations of processing system conditions and optimizing the level of a strong control factor that can counter the variation. Parameter design is carried out in the following procedure [6].

(1) System setting; Implementation of functional design.

(2) Determination of ideal function; determination of ideal function, noise, control factor.

(3) Experimental plan; Assign each factor to the orthogonal table.

(4) Experiments; Experiments were conducted based on assignment of each factor.

(5) Determination of optimal conditions; Calculation of SNR and Sensitivity (SENS). Use the factor effect diagram to obtain the optimum condition.

(6) Confirmation experiment; Confirm the reliability and reproducibility of the estimated value of the gain obtained under optimum conditions.

5.1 System settings Grasping the system when working on the experiment makes it possible to clarify factor 


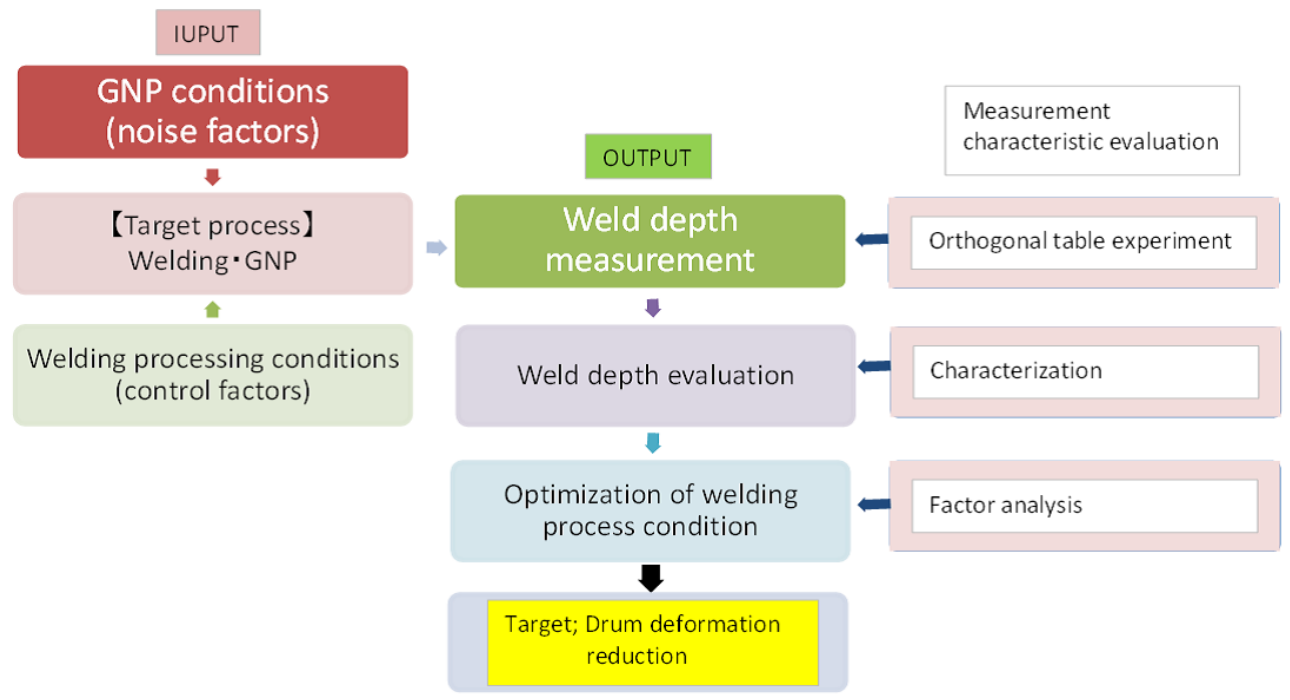

Figure 6: System overview

analysis and classification of factors and to efficiently establish an experimental plan. In quality engineering, the target products and technologies are regarded as systems, and the names of the respective factors are determined, and usually, the output is measured and evaluated. It is clearly distinguished by calling the output as "Measurement characteristic", the factor that causes the output to fluctuate as "Noise factor", and the one inside the system that the engineer can freely decide as "Control factor". Experiment is performed by allocating these parameters to "Orthogonal table". The outline of the system in this research is shown in Fig. 6 [7].

5.2 Determination of Ideal function The aim of this research is to reduce the amount of distortion at the joint and stabilize the quality due to the influence of penetration thermal energy applied at the time of electron beam welding joining of drum and hub. The selection method of the electron beam welding conditions is as follows.

$$
h_{p} \propto \frac{P}{\sqrt{V}}
$$

Weld depth : $h_{p}(\mathrm{~mm})$

Beam output : $P(\mathrm{~kW})$

Welding speed : $V(\mathrm{~m} / \mathrm{min})$

The mechanism of occurrence of strain is shown in Fig. 7. After welding, the drum is distorted to warp up. In order to minimize this amount of distortion, it is important to suppress variations in the shape and depth of the bead portion [1].

Fig. 8 shows the ideal functional state of this system. We set the ideal function state that the welding depth in this system is proportional to the input factor with the welding depth as the evaluation characteristic. By stabilizing the proportionality constant $\beta$ in the figure, it is possible to reduce the drum deformation amount.

Table 1 shows various factors and level tables of this system. For the control factors, welding processing conditions

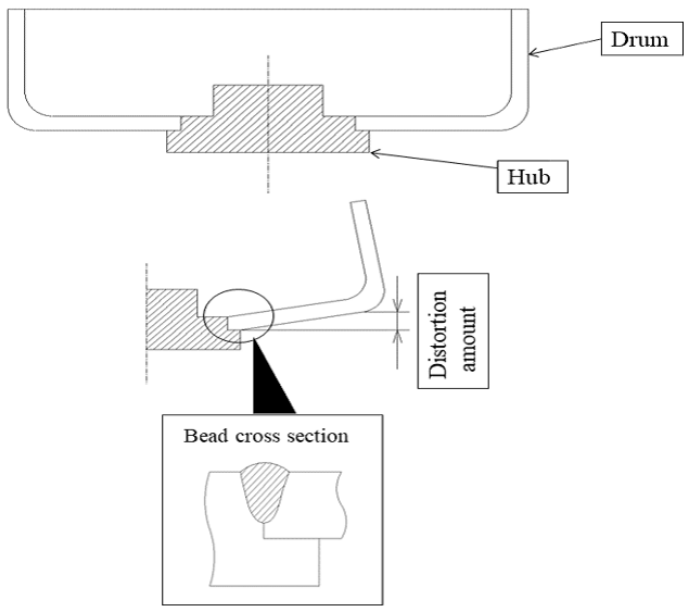

Figure 7: Distortion generation mechanism

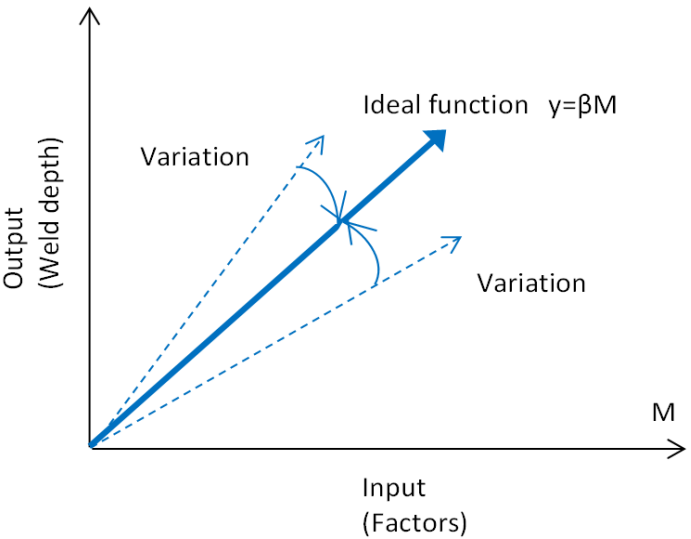

Figure 8: Ideal function

(EBW) were assigned to each level. Since the conditions are restricted by securing the drum surface modification (surface hardness) after welding, the noise factors were set as the condition of the surface reforming treatment (GNP). From this level table, "orthogonal table" which is a statis- 
Table 1: Control and Noise factors level

\begin{tabular}{l|c|c|c|c}
\hline Parameters & Symbol & level 1 & level 2 & level3 \\
\hline Beam mode & A & 8 & 4 & - \\
Spring load & B & small & medium & large \\
(1)Rotation speed (rpm) & C & 8 & 9.5 & 11 \\
(1)Beam current (mA) & D & 35 & 40 & 45 \\
(2) Rotation speed (rpm) & E & 8.5 & 10 & 11.5 \\
(2)Beam current (mA) & F & 70 & 80 & 90 \\
Angle (degree) & G & 35 & 40 & 45 \\
Convergent current (mA) & H & 685 & 690 & 695 \\
\hline
\end{tabular}

\begin{tabular}{c|c}
\hline N1 & N2 \\
\hline GNP con.1 & GNP con.2 \\
\hline
\end{tabular}

Table 2: Assignment to orthogonal table

\begin{tabular}{c||c|c|c|c|c|c|c|c}
\hline No. & A & B & C & D & E & F & G & H \\
\hline 1 & 8 & small & 8 & 35 & 8.5 & 70 & 35 & 685 \\
2 & 8 & small & 9.5 & 40 & 10 & 80 & 40 & 690 \\
3 & 8 & small & 11 & 45 & 11.5 & 90 & 45 & 695 \\
4 & 8 & medium & 8 & 35 & 10 & 80 & 45 & 695 \\
5 & 8 & medium & 9.5 & 40 & 11.5 & 90 & 35 & 685 \\
6 & 8 & medium & 11 & 45 & 8.5 & 70 & 40 & 690 \\
7 & 8 & large & 8 & 40 & 8.5 & 90 & 40 & 695 \\
8 & 8 & large & 9.5 & 45 & 10 & 70 & 45 & 685 \\
9 & 8 & large & 11 & 35 & 11.5 & 80 & 35 & 690 \\
10 & 4 & small & 8 & 45 & 11.5 & 80 & 40 & 685 \\
11 & 4 & small & 9.5 & 35 & 8.5 & 90 & 45 & 690 \\
12 & 4 & small & 11 & 40 & 10 & 70 & 35 & 695 \\
13 & 4 & medium & 8 & 40 & 11.5 & 70 & 45 & 690 \\
14 & 4 & medium & 9.5 & 45 & 8.5 & 80 & 35 & 695 \\
15 & 4 & medium & 11 & 35 & 10 & 90 & 40 & 685 \\
16 & 4 & large & 8 & 45 & 10 & 90 & 35 & 690 \\
17 & 4 & large & 9.5 & 35 & 11.5 & 70 & 40 & 695 \\
18 & 4 & large & 11 & 40 & 8.5 & 80 & 45 & 685 \\
\hline
\end{tabular}

tical tool of optimization method was created to formulate experimental plan.

5.3 Experiment plan / method Table 2 shows an experimental plan table in which control factors are assigned to the inside and noise factors are allocated to the outside using the L18 orthogonal table. The number of experiments is determined by the scale of the orthogonal table and is represented by the experiment number shown at the left end of the orthogonal table. The number of experiments in this system is $18 \times 2=36$ times, and the upper row in the horizontal direction represents the type of control factor allocated.

The numerical values listed below represent the level of each control factor. The advantage of using an orthogonal table is the reduction in the number of experiments. When the orthogonal table $\mathrm{L} 18$ is not used, $2 \times 37=4,374$ experiment times are required.

5.4 Determination of optimum condition In the parameter design, the scale of the evaluation "SNR" is obtained based on the experimental data, and ultimately the presence or absence of the effect is judged with the chart "factor effect diagram" to obtain the optimum condition. The SNR is an evaluation index of robustness, which is calculated as a signal-to-noise ratio taking the ratio of useful component and harmful component, and it is preferable that the numerical value is large.

There are "static characteristics" and "dynamic characteristics" in the types of quality characteristics, and the static characteristic is a characteristic for examining the output without changing the input, and the target value is a constant quality characteristic. In this study, since the target value is fixed, the static characteristic was applied. There are several kinds of evaluation methods of "SNR" in static characteristics, and a method suitable for the purpose is adopted [9].

Each evaluation method will be described below.

(1) "Preferably small characteristics": With non-negative characteristics, the ideal value is zero, the smaller the better the characteristic value.

(2) "Preferably large characteristics": The ideal value is infinite with nonnegative characteristics, and the larger the better the characteristic value.

(3) "Preferably target characteristics": A target value is a finite value, it is bad even if it is small or large, and it is a better characteristic as it is close to a certain target value.

In this research, reduction of drum deformation amount after welding is a subject, and the target value is finite, so the SNR was calculated by "Preferably target characteristics". A method of calculating the SNR and SENS of the preferably target characteristics will be described below [10].

From the $n$ pieces of data $y_{1}, y_{2}, \cdots, y_{n}$ obtained in the experiment, the variance $\hat{\sigma}^{2}$ which is the square of the mean value $\hat{m}$ and the square of the standard deviation $\hat{\sigma}$ is obtained and the SNR is calculated. The number of data is the number obtained by multiplying the noise factor level number and the experiment iteration number. Data analysis in parameter design is based on mathematics of decomposition of sum of squares. Here, the equation for decomposing the total fluctuation (total sum of squares) of the data into general average fluctuation and error fluctuation according to the decomposition of the sum of squares is shown below [6].

The total variation $S_{T}$;

$$
S_{T}=y_{1}^{2}+y_{2}^{2}+\cdots+y_{n}^{2} \quad(f=n)
$$

Where, $f$ represents degrees of freedom.

The general average variation $S_{m}$;

$$
S_{m}=\frac{1}{n}\left(\sum_{i=1}^{n} y_{i}\right)^{2} \quad(f=1)
$$

The error variation $S_{e}$;

$$
S_{e}=S_{T}-S_{m} \quad(f=n-1)
$$

The error variance $V_{e}$;

$$
V_{e}=\frac{S e}{(n-1)}
$$


Table 3: Experimental data and SNR / SENS

\begin{tabular}{c|c|c|c|c}
\hline & \multicolumn{2}{|c|}{ Output data } & \multirow{2}{*}{$\begin{array}{c}\text { SNR } \\
(\mathrm{db})\end{array}$} & $\begin{array}{c}\text { SENS } \\
(\mathrm{db})\end{array}$ \\
\cline { 2 - 3 } & $\begin{array}{c}\text { N1 } \\
\text { GNP Con.1 }\end{array}$ & $\begin{array}{c}\text { N2 } \\
\text { GNP Con.2 }\end{array}$ & 23.6 & 16.95 \\
\hline \hline 1 & 7.4 & 6.7 & 6.42 & 7.49 \\
2 & 3.3 & 1.7 & 2.19 & 1.46 \\
3 & 2.0 & 0.7 & 11.72 & 9.54 \\
4 & 2.5 & 3.6 & 8.17 & 14.41 \\
5 & 4.0 & 6.9 & 4.85 & 3.42 \\
6 & 2.2 & 1.0 & 27.36 & 27.27 \\
7 & 22.4 & 23.8 & 13.57 & 17.41 \\
8 & 6.4 & 8.6 & 15.18 & 16.77 \\
9 & 6.1 & 7.8 & 27.46 & 18.43 \\
10 & 8.1 & 8.6 & 19.79 & 22.36 \\
11 & 12.2 & 14.1 & 34.01 & 11.00 \\
12 & 3.5 & 3.6 & 11.11 & 18.72 \\
13 & 7.1 & 10.5 & 11.80 & 23.44 \\
14 & 12.4 & 17.8 & -4.24 & 13.66 \\
15 & 6.9 & 5.5 & 12.11 & 18.00 \\
16 & 1.8 & 12.9 & 8.86 & 12.29 \\
17 & 7.6 & 8.3 &
\end{tabular}

SNR $\eta$ is calculated from the following equation.

$$
\eta=10 \log \frac{\hat{m}^{2}}{\hat{\sigma}^{2}}=10 \log \frac{1}{V_{e}} \frac{\left(S_{m}-V_{e}\right)}{n}
$$

The magnitude of the output of the experimental system is represented by an index called "SENS". It is calculated by the following equation which logarithmically transforms the square of the average value and multiplies it by 10 .

SENS of static characteristics;

$$
S=10 \log \hat{m}^{2}=10 \log \frac{1}{n}\left(S_{m}-V_{e}\right)
$$

Analysis of the desired characteristics goes through two steps: robustness (stability) improvement and output tuning. The SNR is used for improving the robustness, and SENS is used in the tuning of the output. Table 3 shows the experimental data and calculation results of SNR and SENS.

5.4.1 Analysis of Factor Effect A graph showing the results of Table 3 in a factorial effect diagram (Response graph) is shown in Fig. 8. The vertical axes in the upper and lower figures represent the values of SNR and SENS, respectively, and the horizontal axis represents factor levels. The meaning of this diagram is judged to be effective for reducing the amount of deformation of the drum tooth

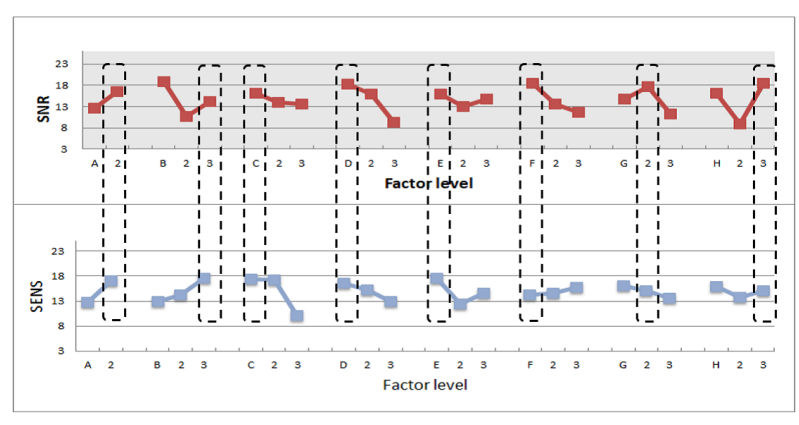

Figure 9: Response graph

width by a control factor which SNR is widened upward and downward. In the static characteristic evaluation, it is desirable that the value of SNR is linearly large, and the optimum condition is determined based on comprehensive evaluation comparing SNR and SENS factor effect diagram [7].

The level judged to be optimal in Fig. 9 is the factor surrounded by the dotted line; "A2: Beam mode 4", "B3: Spring load large", "C1: (1) Rotation speed 8 (rpm)", "D1: (1) Beam current 35 (mA)", "E1: (2) Rotation speed 8.5 (rpm)", "F1: (2) Beam current 70 (mA)", "G2: Angle 40 (degree)", "H3: Convergent current 695 (mA)".

5.4.2 Confirmation of reliability Table 4 shows the calculation results of the SNR estimate in the current condition and the optimum condition selected on the factor effect diagram in previous section. The estimated value of the optimum condition is higher than the current condition. Based on this result, it was confirmed whether the L18 orthogonal table test result is reliable. In the parameter design, the maximum value and the minimum value of the SNR obtained in the orthogonal table experiment are obtained, and if the difference between $10 \%$ of the difference and the estimated SNR is within the range of $10 \%$, it can be judged that there is reliability.

Table 5 shows the evaluation results of reliability. In the evaluation method in this experiment, it was judged that reliability is given to orthogonal table experiment by adopting evaluation of characteristic value (Preferably target characteristics) for a certain target value. This can be said that the optimum condition determined by the parameter design has selected an appropriate level from among several combinations.

Table 4: SNR / SENS estimation

\begin{tabular}{l|c|c|c|c|c|c|c|c|c|c}
\hline \multicolumn{2}{c|}{ Parameter } & $\mathrm{A}$ & $\mathrm{B}$ & $\mathrm{C}$ & $\mathrm{D}$ & $\mathrm{E}$ & $\mathrm{F}$ & $\mathrm{G}$ & $\mathrm{H}$ & Estimate \\
\hline \hline \multirow{3}{*}{$\begin{array}{l}\text { Current } \\
\text { condition }\end{array}$} & Level & 2 & 2 & 2 & 2 & 2 & 2 & 2 & 2 & - \\
\cline { 2 - 12 } & SNR & 16.53 & 10.59 & 13.98 & 15.99 & 12.89 & 13.57 & 17.68 & 8.85 & $\underline{8.46}$ \\
& SENS & 17.08 & 14.22 & 17.18 & 15.20 & 12.48 & 14.66 & 15.07 & 13.74 & 15.24 \\
\hline \multirow{3}{*}{$\begin{array}{l}\text { Optimum } \\
\text { condition }\end{array}$} & Level & $\underline{2}$ & $\underline{3}$ & $\underline{1}$ & $\underline{1}$ & $\underline{1}$ & $\underline{1}$ & $\underline{2}$ & $\underline{3}$ & - \\
\cline { 2 - 10 } & SNR & 16.53 & 14.14 & 16.08 & 18.29 & 15.95 & 18.45 & 17.68 & 18.53 & $\underline{34.04}$ \\
& SENS & 17.08 & 17.57 & 17.43 & 16.57 & 17.62 & 14.25 & 15.07 & 15.12 & 26.32 \\
\hline
\end{tabular}


Table 5: Reliability confirmation

\begin{tabular}{|c|c|c|c|}
\hline \multicolumn{2}{|c|}{ L18 experiment SNR } & \multicolumn{2}{c|}{$\begin{array}{c}\text { Reliability confirmation } \\
\text { by reverse estimation }\end{array}$} \\
\hline Maximum & 18.32 & $\begin{array}{c}\text { Average of } \\
\text { current condition }\end{array}$ & 8.46 \\
\hline Minimun & 8.85 & $\begin{array}{c}\text { Reverse estimated } \\
\text { value }\end{array}$ & 34.04 \\
\hline $10 \%$ of difference & $\underline{0.947}$ & \multicolumn{2}{|}{$\underline{\text { Reliable }}$} \\
\hline \multicolumn{2}{|c|}{ Judgment } & \multicolumn{2}{|c|}{} \\
\hline
\end{tabular}

Table 6: Confirmation experimental data

\begin{tabular}{|c|cc|c|c|}
\hline & $\begin{array}{c}\text { N1 } \\
\text { GNP cond.1 }\end{array}$ & $\begin{array}{c}\text { N2 } \\
\text { GNP cond.2 }\end{array}$ & $\begin{array}{c}\text { SNR } \\
(\mathrm{db})\end{array}$ & $\begin{array}{c}\text { SENS } \\
(\mathrm{db})\end{array}$ \\
\hline Current & 25.7 & 7.5 & 0.66 & 22.85 \\
\hline Optimum & 5.5 & 5 & 23.42 & 14.39 \\
\hline
\end{tabular}

5.5 Confirmation experiment The purpose of the confirmation experiment is to confirm the effect of the selected optimum condition. However, in the parameter design, it is a point to check the difference (gain) from the current condition rather than simply investigate the SNR and SENS under the optimum condition and compare it with the estimated value. In other words, it is a confirmation experiment to examine the reproducibility of the gain, not the reproducibility of the absolute values of SNR and SENS [3].

In the experimental procedure, firstly, the estimated value of the SNR of the selected optimum condition and the current condition is obtained, and experiments are conducted again on the same two types as the orthogonal table experiment. Next, the SNR is calculated from the experimental results, and the difference between the estimated values of both of them is compared with the difference (gain) in the confirmation experiment, and it is investigated whether or not the experiment is reproducible.

Table 6 shows the confirmation experiment data, and Table 7 shows the evaluation results of reproducibility. The condition of reproducibility in the confirmation experiment in the parameter design is that the difference between the estimated value of the optimum condition and the gain is within $\pm 30 \%$. The reason for judging reproducibility by gain is that the improvement effect of the control factor can be consistently reproduced even if the environment is different. Therefore, if the difference between the estimated value and the gain of the optimum condition is within the range of $\pm 30 \%$, it is hard to be affected by the interaction and it can be judged that reproducibility of the experimental effect can be obtained.

Furthermore, from the statistical empirical rule, if the experimental value is within $\pm 3 \mathrm{db}$ for the estimated value, it can be judged that there is reproducibility. Therefore, from the results in Table $7(|89-68|= \pm 21 \% \leqq \pm 30 \%$, $|25.57-22.76|= \pm 2.81 \mathrm{db} \leqq \pm 3 \mathrm{db})$, it can be judged that the present experiment is totally reproducible [3] [11].

\section{Conclusion}

The results of this research are as follows.

(1) By optimizing the system, the quality was improved
Table 7: Reproducibility of gain

\begin{tabular}{|c|c|c|c|}
\hline \multirow{2}{*}{} & \multicolumn{3}{|c|}{ SNR } \\
\cline { 2 - 4 } & Estimate & Conf. value & Reproducibility \\
\hline Current & 8.46 & 0.66 & $8 \%$ \\
Optimum & 34.40 & 23.42 & $\underline{68 \%}$ \\
\hline Gain & $\underline{25.57}$ & $\underline{22.76}$ & $\underline{89 \%}$ \\
\hline \multicolumn{2}{|c|}{ Difference of gain (db) } & $\underline{2.81}$ & $\underline{\text { Judge : OK }}$ \\
\hline
\end{tabular}

more than before, and the variation range of the drum tooth width deformation amount was reduced as compared with the existing machining conditions. (Reduction width : $5.1 \mu \mathrm{m}$, rate : $54 \%$ reduction)

(2) The process capability was improved by reducing the variation range of the drum tooth width deformation amount. $\left(C_{p}=1.79>1.33\right)$

(3) With the improvement of process capability, the manufacturing cost was reduced by $30 \%$ due to the abolition of the $100 \%$ inspection of all products after processing.

The evaluation result of "process capability" which is the outcome indicator of this research is described below. "Process capability" refers to the ability to express the probability of falling within the product's quality standard in making a product. "Process capability index $\left(C_{p}\right)$ " is used as a measure indicating the degree of stability of the quality characteristic due to process variation with respect to the width of the standard. Based on the capacity index, grasp the product loss and determine the frequency of inspection. That is, $C_{p}=$ (upper limit standard - lower limit standard) $/ 6 \sigma(\sigma=$ standard deviation). If $C_{p} \geqq 1.33$, the process power difference is sufficient. It is almost satisfactory if $1 \leqq C_{p}<1.33$. If $C_{p}<1$, it is judged that the process capability is insufficient.

Table 8 shows the evaluation results of $C_{P}$ for current conditions and optimum conditions in this research. The number of samples evaluated is 20 , and the calculation method of $C_{P}$ is given by the following equation.

$$
C_{p}=\frac{U S L-L S L}{6 \sigma}
$$

USL:Upper limit specification value

LSL:Lower limit specification value $\sigma:$ standard deviation

STW:Standard width

$$
\sigma=\sqrt{\frac{1}{n} \sum_{n=1}^{n}\left(x_{i}-\bar{x}\right)^{2}}
$$

$n:$ Total number of data

$x_{i}:$ Individual data, $\bar{x}$ :Average value (AVG.)

Fig. 10 and 11 show histograms obtained by summarizing the data distribution (scattered) of $C_{p}$ calculated in Table 8 in the frequency distribution table. From the results 
Table 8: Process capability index

\begin{tabular}{|c|c|}
\hline \multicolumn{2}{|c|}{ Current } \\
\hline Sample & Data \\
\hline 1 & 5.82 \\
\hline 2 & 5.80 \\
\hline 3 & 5.81 \\
\hline 4 & 5.77 \\
\hline 5 & 5.78 \\
\hline 6 & 5.80 \\
\hline 7 & 5.76 \\
\hline 8 & 5.82 \\
\hline 9 & 5.83 \\
\hline 10 & 5.76 \\
\hline 11 & 5.81 \\
\hline 12 & 5.80 \\
\hline 13 & 5.81 \\
\hline 14 & 5.79 \\
\hline 15 & 5.83 \\
\hline 16 & 5.75 \\
\hline 17 & 5.74 \\
\hline 18 & 5.84 \\
\hline 19 & 5.79 \\
\hline 20 & 5.85 \\
\hline AVG. & 5.80 \\
\hline$\sigma$ & 0.03 \\
\hline$C_{p}$ & 1.09 \\
\hline$\overline{\mathrm{Cpk}}$ & 1.07 \\
\hline STW. & 0.20 \\
\hline USL. & 5.90 \\
\hline LSL. & 5.70 \\
\hline
\end{tabular}

\begin{tabular}{|c|c|}
\multicolumn{3}{c}{ Optimum } \\
\hline Sample & Data \\
\hline 1 & 5.79 \\
2 & 5.78 \\
3 & 5.80 \\
4 & 5.79 \\
5 & 5.78 \\
6 & 5.76 \\
7 & 5.74 \\
8 & 5.75 \\
9 & 5.79 \\
10 & 5.78 \\
11 & 5.79 \\
12 & 5.75 \\
13 & 5.76 \\
14 & 5.80 \\
15 & 5.81 \\
16 & 5.77 \\
17 & 5.77 \\
18 & 5.79 \\
19 & 5.78 \\
20 & 5.77 \\
\hline AVG. & 5.78 \\
$\sigma$ & 0.02 \\
$C_{p}$ & 1.79 \\
\hline Cpk & 1.39 \\
STW. & 0.20 \\
USL. & 5.90 \\
LSL. & 5.70 \\
\hline & \\
\hline
\end{tabular}

of the graph, it can be seen that the fluctuation range of the drum tooth width deformation amount is reduced by optimization, thereby improving the process capability.

\section{Future research subject}

Even in electron beam welding, various defects are generated as with other welding methods. In the electron beam welding, when the material and the beam intensely interact in a short period of time, the molten pool generates characteristic porosity and cracks due to a characteristic shape called keyhole type [1] [2].

\section{(1)Porosity}

Beam welding with high energy density generally has a large penetration depth and width aspect ratio so that the molten pool tends to be unstable and the gas trap generated from the atmosphere or molten metal causes a relatively large irregular porosity is generated.

\section{(2) Crack}

High temperature cracking tends to occur at the coagulated grain boundary and at the association part of columnar crystals due to the shape factor of the keyhole type penetration shape and the metallurgical factor that the solidification rate is fast.

With regard to quality problems due to such welding defects, we will work on research to stabilize and optimize the molten pool. Specifically, we plan to optimize welding

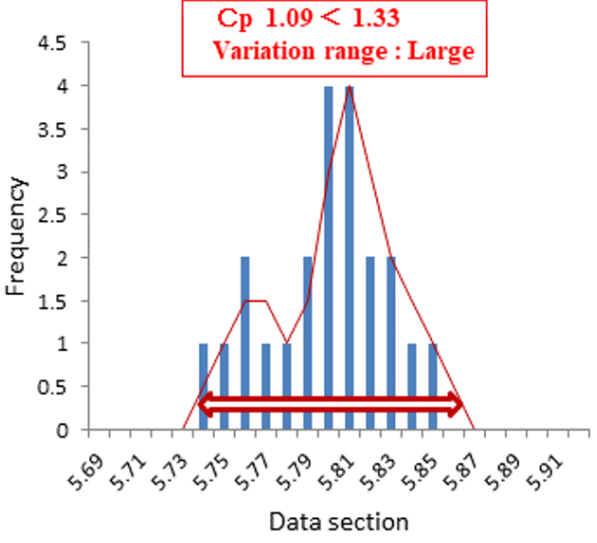

Figure 10: Histogram of Current

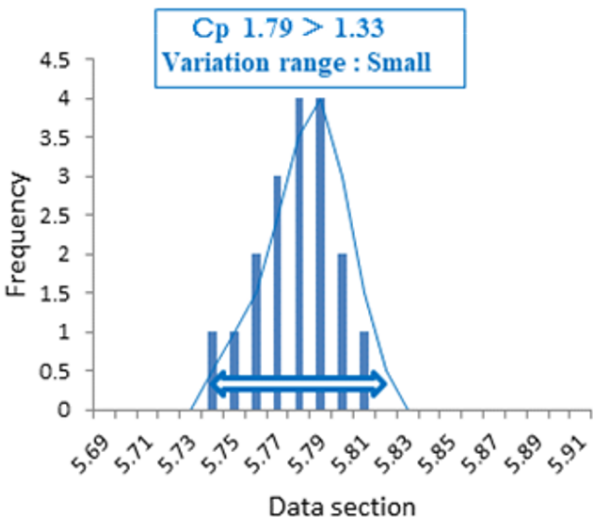

Figure 11: Histogram of Optimum

strength by robust design with parameters such as shape factor such as keyhole penetration, metallurgical factor of solidification rate, material with low crack susceptibility, etc. as parameters.

\section{References}

[1] H. Murakami, T. Ishimi and M. Yasunaga, Welding Society 46th Electron Beam Welding Research Committee Document, EBW-366-85, 1985.

[2] F. Kaneko, H. Saitou and F. Shibata, "Weldability of state graphite cast iron and carbon steel by electron beam welding", Proceedings of the 62nd Annual Meeting of the Japan Society for Materials Science lecture meeting, pp.389-390, 2013.

[3] G. Taguchi, "Evaluation technology for optimization design”, Japan Standards Association, pp.13-90, 2002.

[4] K. Tatebayashi, "Introductory Taguchi Method", Japan Science and Technology Unit, pp.193-249, 2015.

[5] M. Suzuki, "Not difficult quality engineering", Nikkan Kogyo Shimbun, pp.34-48, 2016.

[6] S. Koshimizu, M. Suzuki, "Practical quality engineering", Nikkan Kogyo Shimbun, pp.3-56, 2007.

[7] K. Hirose, T. Ueda, "Introduction to Taguchi Method Analysis Method”, DOYUKAN, pp.1-24, 2015. 
[8] T. Yamamori, N. Shibayama and I. Amamiya, "Automatic transmission [Structure, operation and control]", SANKAIDO, pp.14-55, 2005.

[9] H. Yano, "Introduction to Quality Engineering Calculation Method”, Japan Standards Association, pp.74-137, 2002.

[10] G. Taguchi, H. Yano, "Technical development of machinery, materials and processing", Japan Standards Association, pp150-155, 2001.

[11] H. Yano, "Introduction to Quality Engineering, Japan Standards Association", pp.109-122, 2011.

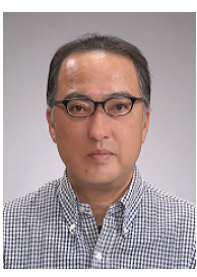

Toma Eiji (Member) was born in Hokkaido, Japan, on December 17, 1959. When he was engaged as a manufacturing engineer at an automobile parts manufacturer, he acquired a "Professional Engineer (P.E.jp)" and "APEC engineer” in 2018, and is presently an professor at National Institute of Technology, Tomakomai College. He ing. He is member of IIAE.

has worked on research of the quality engineer- 